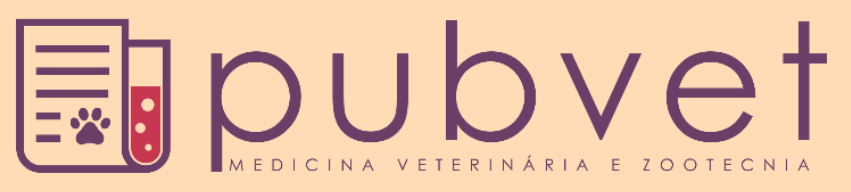

https://doi.org/10.31533/pubvet.v14n1a485.1-6

\title{
Prevalência de endoparasitas em Progne chalybea no dormitório em Chapecó, Santa Catarina, Brasil
}

\author{
Mariléa Fátima Matiazzo $^{1 \bullet}$, Gustavo Dalavale ${ }^{1 \bullet}$, Jackeline Karsten Kirinus ${ }^{2 *} \bullet$ \\ ${ }^{1}$ Discentes de Medicina Veterinária e integrantes do Grupo de Estudo, Pesquisa e Extensão em Sanidade Animal da Unidade \\ Central de Educação FAEM Faculdades. Chapecó, Santa Catarina, Brasil. \\ ${ }^{2}$ Docente da Universidade do Estado de Santa Catarina (UDESC_CEO). Chapecó, Santa Catarina, Brasil. \\ *Autor para correspondência, E-mail: jackeline.kirinus@gmail.com
}

\begin{abstract}
Resumo. Por vários anos a andorinha-grande (Progne chalybea) utiliza como dormitório coletivo o centro da cidade de Chapecó no período que antecede a migração, oportunidade única para se obter dados ecológicos da espécie. A aproximação de aves de vida livre com o ser humano pode ser uma preocupação devido a possibilidade de transmissão de patógenos, assim como, as aves podem ser contaminadas por patógenos humanos ou de animais domésticos. Esse estudo verificou a prevalência de endoparasitas nas amostras fecais de $P$. chalybea, coletadas no dormitório, no período de abril a maio de 2019. Os dados preliminares sugerem o parasitismo por Capillaria e coccídeos (oocistos sugestivos dos gêneros Eimeria e/ou Isospora). Sabe-se que a ocorrência de endoparasitos pode ser utilizada como parâmetro sanitário. Portanto, é importante a continuidade dos trabalhos a fim de determinar quais espécies de parasitos a $P$. chalybea possui. Ademais, estes dados podem ser utilizados em inferências de saúde das aves, visto que elas prestam um importante serviço ecológico no controle de insetos vetores.
\end{abstract}

Palavras chave: andorinha-grande, Capillaria, Eimeria, Isospora

\section{Prevalence of endoparasites in Progne chalybea at dormitory in Chapecó, Santa Catarina, Brazil}

Abstract. For several years, the great swallow (Progne chalybea) has used the collective dormitory of the city center of Chapecó in the period before migration, a unique opportunity to obtain ecological data of the species. The approach of freeliving birds to humans may be a concern due to the possibility of pathogen transmission, as birds may be contaminated by human or domestic pathogens. This study verified the prevalence of endoparasites in the fecal P. chalybea samples collected in the dormitory from April to May 2019. Preliminary data suggest parasitism by Capillaria and coccidia (oocysts suggestive of the genera Eimeria and/or Isospora). It is known that the occurrence of endoparasites can be used as a sanitary parameter. Therefore, further work is important to determine which species of parasites has $P$. chalybea. Moreover, these data can be used in inferences of bird health, since they provide an important ecological service in the control of insect vectors.

Keywords: Great swallow, Capillaria, Eimeria, Isospora 


\title{
Prevalencia de endoparásitos en Progne chalybea en dormitorio en Chapecó, Santa Catarina, Brasil
}

\begin{abstract}
Resumen. Durante varios años, la Gran golondrina (Progne chalybea) ha utilizado el dormitorio colectivo del centro de la ciudad de Chapecó en el período anterior a la migración, una oportunidad única para obtener datos ecológicos de la especie. El enfoque de las aves de vida libre a los humanos puede ser una preocupación debido a la posibilidad de transmisión de patógenos, ya que las aves pueden estar contaminadas por patógenos humanos o domésticos. Este estudio verificó la prevalencia de endoparásitos en las muestras fecales de $P$. chalybea recolectadas en el dormitorio de abril a mayo de 2019. Los datos preliminares sugieren parasitismo por Capillaria y coccidios (oocistos que sugieren los géneros Eimeria y/o Isospora). Se sabe que la aparición de endoparásitos se puede utilizar como parámetro sanitario. Por lo tanto, el trabajo adicional es importante para determinar qué especies de parásitos tiene $P$. chalybea. Además, estos datos pueden usarse para inferir la salud de las aves, ya que proporcionan un servicio ecológico importante para el control de vectores de insectos.
\end{abstract}

Palabras clave: Gran golondrina, Capillaria, Eimeria, Isospora

\section{Introdução}

A Progne chalybea (andorinha-grande) (Gmelin, 1789) é uma espécie de ave pertencente à família Hirundinidae, ordem Passeriformes, que possui 16 espécies ocorrentes no Brasil (Piacentini et al., $\underline{2015}$ ), sendo $P$. chalybea a maior de todas medindo $19,5 \mathrm{~cm}$ de comprimento (Sick, 1997). Essa ave possui registro de ocorrência do México à Argentina (Sick, 1997) em áreas rurais e urbanas; porém, evita áreas florestadas (Sigrist, 2009). É considerada uma migrante neotropical, que se reproduz no sul do Brasil (Eisenmann \& Haverschmidt, 1970) e migra para o norte da América do Sul durante o período não reprodutivo (maio a setembro) (Naka et al., 2001).

A migração pode ser considerada uma adaptação de algumas espécies para enfrentar a escassez sazonal de recursos, além de explorar sua abundância em outras regiões (Fernandes et al., 2007). A $P$. chalybea é especializada na captura de insetos voadores na coluna de ar (Ramstack et al., 1998) e migra em busca da maior abundância desse recurso alimentar (Fernandes et al., 2007).

Há vários anos, no período que antecede a migração, milhares de indivíduos dessa espécie agrupam-se no centro da cidade de Chapecó, onde utilizam árvores, edificações e o sistema da rede elétrica para pernoite.

Neste sentido, a aproximação das aves de vida livre com o ser humano (Zanella, 2016) pode contribuir para a transmissão de doenças, em função desses animais, por vezes, servirem de hospedeiros ou reservatórios para patógenos com potenciais zoonóticos, como por exemplo parasitos (Torres et al., 2015). Por outro lado, inverso também é possível, onde as aves podem contaminar-se durante o convívio humano. Nesse contexto, é importante a realização de estudos a fim de contribuir na prevenção de danos à saúde pública (Snak et al., 2014; Sousa et al., 2019), além de obter-se dados da qualidade ecológica do ambiente onde essas aves vivem.

O objetivo dessa pesquisa foi verificar a prevalência de endoparasitos em amostras fecais do dormitório da P. chalybea (andorinha-grande) no centro da cidade de Chapecó, Santa Catarina, Brasil.

\section{Material e métodos}

Foram coletados 30 pools de amostras de fezes do grupo de andorinhas da espécie $P$. chalybea no dormitório localizado na avenida Getúlio Dornelles Vargas esquina com a rua Marechal Deodoro no centro da cidade de Chapecó, Santa Catarina, Brasil ( $27^{\circ} 06^{\prime} 00^{\prime \prime S}$; 52 $\left.32^{\circ} 36^{\prime} 56^{\prime \prime W}\right)$ no período de abril a maio de 2019. Para a coleta foi estendida uma lona nova de quatro metros quadrados (asséptica) abaixo das árvores utilizadas para o repouso das aves. Esta lona foi colocada às $22 \mathrm{~h} 00 \mathrm{e}$ as amostras foram recolhidas às $05 \mathrm{~h} 30$ do dia seguinte. O local do dormitório já era isolado da circulação de pessoas pela Secretaria de Serviços Urbanos do Município de Chapecó. 
As amostras foram transportadas em caixa de isopor isotérmica com gelo reciclável e encaminhadas até o laboratório de Parasitologia da Unidade Central de Educação FAEM Faculdades (UCEFF Chapecó) onde foi realizado o processamento das mesmas.

Cada uma das amostras foi submetida aos métodos de análises qualitativas de flutuação, Técnica de Willis (1921) modificada e sedimentação por centrifugação, descritas por Monteiro (2011) com o objetivo de localizar ovos de helmintos e cistos ou oocistos de protozoários.

\section{Resultados e discussão}

No pool de amostras coletadas foram identificados nematódeos da família Trichuridae, gênero Capillaria e protozoários da família Eimeriidae (oocistos sugestivos dos gêneros Eimeria e/ou Isospora).

Estudos realizados em aves citam algumas espécies de parasitos em $P$. chalybea, como os pertencentes aos gêneros Lutztrema (Trematoda) (Travassos, 1941) e Diplotriaena (Nematoda) (Pinto \& Noronha, 1971; Travassos et al., 1927; Vicente et al., 1983). Esse último gênero possui especificidade parasitária restrita as aves e com marcada preferência pelas insetívoras (Vicente et al., 1983). Nenhum desses parasitos foi observado nas amostras desta pesquisa.

Os nematóides são considerados os parasitos mais patogênicos e comumente observados em aves silvestres (Rennó et al., 2008), tendo como representantes o gênero Capillaria (identificado nesse estudo para $P$. chalybea). Estes são considerados vermes de distribuição cosmopolita que afetam inúmeras espécies da ordem Passeriformes (Ritchie et al., 1997), aves de companhia e de produção; porém são mais comuns em araras, periquitos, canários, pombos e galináceos (Ritchie et al., 1997). Ademais, estes parasitos são encontrados especialmente naqueles animais mantidos em ambientes contaminados e de alta densidade populacional, como zoológicos e criadouros (Ayres et al., 2016).

A transmissão destes parasitos ocorre pela liberação, no ambiente, dos ovos nas fezes dos animais contaminados, conforme verificado na amostra de material fecal de $P$. chalybea. Estes ovos podem permanecer no ambiente durante meses, em condições adequadas de umidade e temperatura, e posteriormente são ingeridos através de água e alimentos contaminados, o que pode ocasionar o parasitismo nas aves (Rennó et al., 2008). Ademais, a dieta alimentar não parece estar associada a suscetibilidade e pode afetar tanto granívoros, onívoros como insetívoros; no entanto, pode envolver minhocas como hospedeiros paratênicos (Ritchie et al., 1997), os quais são mais comuns na alimentação de onívoros.

Nestes estudos, foram identificadas várias espécies do gênero Capillaria parasitando aves (Hofstatter \& Guaraldo, 2015; Marietto-Gonçalves et al., 2009; Ritchie et al., 1997; Vicente et al., 1997). Contudo, até o momento, nenhum trabalho citou o gênero parasitando a $P$. chalybea. Dentre os protozoários analisados, os coccídeos do gênero Eimeria e Isospora são mencionados como os de maior importância em medicina veterinária (Monteiro, 2011) e são considerados os parasitos intestinais mais comuns em aves (Lopez et al., 2007; Vasconcelos et al., 2012). Em espécies silvestres, a maior prevalência de parasitismo por coccídeos é em Passeriformes (Berto et al., 2011; Marietto-Gonçalves et al., 2009), na qual está inclusa $P$. chalybea.

Em um estudo com andorinhas migratórias realizado por Fakhar et al. (2018) foi identificado coccídeos parasitando Hirundo rustica Linnaeus, 1758 andorinha-de-bando, mas sem determinação de gênero e espécie. Tais resultados corroboram com o encontrado neste trabalho, onde sugere-se que os oocistos não esporularam e desta forma não foi possível sua identificação, muito embora pesquisas apontem que em Passeriformes as infecções são principalmente por espécies pertencentes ao gênero Isospora (Berto et al., 2011; Lopez et al., 2007; Ritchie et al., 1997).

Em função da variação circadiana de liberação dos oocistos pelos Passeriformes, as técnicas mais comuns de detecção e quantificação de coccídeos podem ser imprecisas para estudos de campo, o que sugere concentrar as coletas no período da tarde (Lopez et al., 2007), fato importante a ser considerado em amostragens para estudos futuros.

Para hirundinídeos do Novo Mundo foi descrito o coccídeo Isospora petrochelidon (Stabler \& Kitzmiller, 1972) parasitando Petrochelidon pyrrhonota (Vieillot, 1817) andorinha-de-dorso-acanelado (Berto et al., 2011). Essa espécie de andorinha é visitante sazonal no Brasil vinda do Hemisfério Norte (Piacentini et al., 2015) e pode propagar esse patógeno em sua rota de migração, visto que pode visitar 
as mesmas áreas que $P$. chalybea. É importante que na continuação deste estudo seja determinado qual espécie de coccídeo parasita a andorinha-grande.

O grupo das aves é bastante estudado para endoparasitos, seja pela sua diversidade, relativa facilidade na obtenção de amostras ou em função da sua ampla distribuição nos ecossistemas. Mesmo que a maior prevalência de parasitismo seja em aves cativas que em aves de vida livre, possivelmente este fato pode refletir um manejo inadequado do plantel (Marietto-Gonçalves et al., 2009). Assim, faz-se necessário novos estudos que pesquisem a ocorrência de endoparasitos nestas aves, em função delas serem consideradas indicadoras da qualidade de um ambiente. Além disso, as informações com aves de vida livre podem ser utilizadas como parâmetro sanitário e quando avalia-se espécies migratórias (como $P$. chalybea) é possível fazer inferências em mais de um ambiente ao longo de sua rota (Costa et al., 2010).

Nas aves de vida livre, em alguns casos, o parasitismo pode estar diretamente relacionado ao hábito alimentar do hospedeiro, onde espécies onívoras e granívoras podem ser mais parasitadas em comparação às aves insetívoras (Costa et al., 2010). Por outro lado, as aves granívoras e onívoras ampliam as formas de contágio durante o consumo de frutos ou durante a coleta de sementes no solo; ao contrário das insetívoras, como no caso de $P$. chalybea, que ao capturar insetos em voo podem limitarse ao risco desses como vetores para o parasitismo. Assim, a $P$. chalybea, essencialmente insetívora, é responsável por prestar um importante serviço ecológico no controle de insetos que podem ser vetores de doenças aos humanos e outras espécies de mamíferos mantidos como animais domésticos.

Portanto, faz-se importante a continuação dos trabalhos com $P$. chalybea, visto esta ser considerada uma espécie migratória e que possui dormitório em área urbana com grande circulação de pessoas, a fim de investigar a prevalência de parasitos ao longo dos anos e a informação que trazem dos ambientes que elas frequentam. $O$ fato de conhecer o período em que estas aves fazem uso do local para descanso e o tempo de permanência neste ambiente facilita a realização das amostragens. Além disso, o comportamento gregário que antecede a migração, quando milhares de indivíduos compartilham o mesmo dormitório, propicia a propagação de patógenos entre elas, caso o possuam, o que torna essa espécie interessante para estudo.

Ressalta-se para um fator imprescindível, ou seja, que a Secretaria de Serviços Urbanos do Município de Chapecó continue a isolar a área utilizada como dormitório por $P$. chalybea, além de instalar lonas na calçada todas as noites e lavar o local ao amanhecer, o que minimiza o odor deixado pelas fezes e evita que a população transporte os resíduos nos calçados. Estas medidas representam o cuidado com a natureza, uma das frentes para uma cidade sustentável.

\section{Agradecimentos}

Especial agradecimento à Adriane Siqueira, Ana Maria Salvagni, Cintia Carasek, Eduarda Olivette, Guilherme Oselame, Nadine Cristiane Henz, Sabrina Majolo e Valeria Oro pelo auxílio na coleta e processamento das amostras e ao MercoLab Laboratórios de Chapecó pelo apoio nas análises laboratoriais.

\section{Referências bibliográficas}

Ayres, M. C. C., Peixoto, M. S. R., Silva, W. B., Gomes, D. M., Nunes, O. C., Borges, K. B., . . . Almeida, M. A. O. (2016). Ocorrência de parasitos gastrintestinais em Psitacídeos, mantidos em Parques Ecológicos na região metropolitana de Salvador, Bahia. Brazilian Journal of Veterinary Medicine, 38(2):133-136.

Berto, B. P., Flausino, W., McIntosh, D., Teixeira-Filho, W. L. \& Lopes, C. W. G. (2011). Coccidia of new world passerine birds (Aves: Passeriformes): a review of Eimeria Schneider, 1875 and Isospora Schneider, 1881 (Apicomplexa: Eimeriidae). Systematic Parasitology, 80(3):159-204.

Costa, Í. A., Coelho, C. D., Bueno, C., Ferreira, I. \& Freire, E. (2010). Ocorrência de parasitos gastrintestinais em aves silvestres no município de Seropédica, Rio de Janeiro, Brasil. Ciência Animal Brasileira, 11(4):914-922.

Eisenmann, E. \& Haverschmidt, F. (1970). Northward migration to Surinam of South American martins (Progne). The Condor, 72(3):368-369. 
Fakhar, M., Chegeni, T. N., Bastani, R., Hosseininejad, Z., Saberi, R. \& Armat, S. (2018). Intestinal parasites among migrant barn swallows (Hirundo rustica) in the central region of Mazandaran Province, Northern Iran. Veterinary World, 11(8):1179-1182.

Fernandes, F. R., Cruz, L. D. \& Rodrigues, A. A. F. (2007). Diet of the Gray-Breasted Martin (Hirundinidae: Progne chalybea) in a wintering area in Maranhão, Brazil. Revista Brasileira de Ornitologia, 15445-447.

Hofstatter, P. G. \& Guaraldo, A. M. A. (2015). Parasitological survey on birds at some selected Brazilian zoos. Revista Brasileira de Parasitologia Veterinária, 24(1):87-91.

Lopez, G., Figuerola, J. \& Soriguer, R. (2007). Time of day, age and feeding habits influence coccidian oocyst shedding in wild passerines. International Journal for Parasitology, 37(5):559-564.

Marietto-Gonçalves, G. A., Martins, T. F., Lima, E. T., Souza Lopes, R. \& Andreatti Filho, R. L. (2009). Prevalência de endoparasitas em amostras fecais de aves silvestres e exóticas examinadas no Laboratório de Ornitopatologia e no Laboratório de Enfermidades Parasitárias da FMVZUNESP/Botucatu-SP. Ciência Animal Brasileira, 10(1):349-354.

Monteiro, S. G. (2011). Parasitologia na medicina veterinária (Vol. 1). São Paulo: Roca.

Naka, L. N., Rodrigues, M., Roos, A. L. \& Azevedo, M. A. G. (2001). Bird conservation on Santa Catarina Island, Southern Brazil. Bird Conservation International, 12(2):123-150.

Piacentini, V. Q., Aleixo, A., Agne, C. E., Maurício, G. N., Pacheco, J. F., Bravo, G. A., . . Posso, S. (2015). Annotated checklist of the birds of Brazil by the Brazilian Ornithological Records Committee. Revista Brasileira de Ornitologia, 23(2):91-298.

Pinto, R. M. \& Noronha, D. (1971). Sobre dois novos filarideos parasitas de andorinha doméstica (Nematoda, Filarioidea). Atas Soco Biologia, 14(5-6):121-123.

Ramstack, J. M., Murphy, M. T. \& Palmer, M. R. (1998). Comparative reproductive biology of three species of swallows in a common environment. The Wilson Bulletin, 110233-243.

Rennó, P. P., Queiroz, F. M., Garcia, B. P., Prado, R. N. A., Simões, M. M., Souza, J. P. F., . . Pereira, R. E. P. (2008). Endoparasitose em aves-revisão de literatura. Revta Científica Eletrônica de Medicina Veterinária, 6(11):1-6.

Ritchie, B. W., Hsarrison, G. J., Zantop, D. \& Harrison, L., R,. (1997). Avian medicine: principles and application, abridged edition. USA: Idaho Falls, ID: Wingers Publishing.

Sick, H. (1997). Ornitologia Brasileira. Rio de Janeiro, Brasil: Nova Fronteira.

Sigrist, T. (2009). Iconografia das aves do Brasil: Bioma cerrado (Vol. 1). São Paulo, Brasil: Avis Brasilis.

Snak, A., Lenzi, P. F., Agostini, K. M., Delgado, L. E., Montanucci, C. R. \& Zabott, M. V. (2014). Análises coproparasitológicas de aves silvestres cativas. Ciência Animal Brasileira, 15(4):502-507.

Sousa, T. N., Silva, R. V. S., Evangelista, B. B. C. \& Freire, S. M. (2019). Prevalência das zoonoses parasitárias e a sua relação com as aves silvestres no nordeste do Brasil. Jornal Interdisciplinar de Biociências, 3(2):39-44.

Stabler, R. M. \& Kitzmiller, N. J. (1972). Isospora petrochelidon sp. n. (Protozoa: Eimeriidae) from the cliff swallow, Petrochelidon pyrrhonota. The Journal of Protozoology, 19(2):248-251.

Torres, A. C. D., D'Aparecida, N. S. \& Haas, D. J. (2015). Principais zoonoses víricas, fúngicas e parasitárias de aves domésticas e silvestres. Veterinária em Foco, 13(1):44-55.

Travassos, L. (1941). Lutztrema ng: (Trematoda: Dicrocoeliidae). Memórias do Instituto Oswaldo Cruz, 36(3):335-343.

Travassos, L., Pinto, C. \& Muniz, J. (1927). Excursão cientifica ao Estado de Matto Grosso na zona do Pantanal (Margens dos Rios S. Lourenço e Cuyabá) realisada em 1922. Memórias do Instituto Oswaldo Cruz, 20(2):249-269.

Vasconcelos, T. C. B., Silva Longa, C., Campos, S. D. E., Costa, C. H. C. \& Bruno, S. F. (2012). Coccidiose em Sporophila maximiliani (Passeriformes: Emberizidae): relato de dois casos. Brazilian Journal of Veterinary Medicine, 34(4):261-264. 
Vicente, J. J., Pinto, R. M. \& Noronha, D. (1983). Estudo das espécies brasileiras do gênero Diplotriaena Henry \& Ozoux, 1909 (Nematoda, Filarioidea). Memórias do Instituto Oswaldo Cruz, 78165-182.

Vicente, J. J., Rodrigues, H. O., Gomes, D. C. \& Pinto, R. M. (1997). Nematóides do Brasil. Parte V\&58; nematóides de mamíferos Brazillan nematodes. Part V\&58; nematodes of mammals. Revista Brasileira de Zoologia, 14(1):1-452.

Willis, H. H. (1921). A simple levitation method for the detection of hookworm ova. Medical Journal of Australia, 2(18):375-376.

Zanella, J. R. C. (2016). Zoonoses emergentes e reemergentes e sua importância para saúde e produção animal. Pesquisa Agropecuária Brasileira, 51(5):510-519.

Recebido: 3 de novembro, 2019.

Aprovado: 1 de dezembro, 2019.

Publicado: 29 de fevereiro, 2020.

Licenciamento: Este artigo é publicado na modalidade Acesso Aberto sob a licença Creative Commons Atribuição 4.0 (CC-BY 4.0), a qual permite uso irrestrito, distribuição, reprodução em qualquer meio, desde que o autor e a fonte sejam devidamente creditados. 